\title{
COVID-19 E ERITEMA NODOSO: UM RELATO DE CASO
}

\author{
COVID-19 AND ERYTHEMA NODOSUM: A CASE REPORT
}

\author{
Geórgia Mansur ${ }^{1}$ \\ Bernardo Oliveira Castro de Azevedo Oliveira ${ }^{2}$ \\ Amanda Lívia Silva Moura ${ }^{3}$ \\ Tiago Lucas Tadeu Carvalho Castro ${ }^{4}$ \\ Diogo Lemos Araújo ${ }^{5}$ \\ Guilherme Henrique Machado ${ }^{6}$
}

RESUMO: A pandemia pelo SARS-Cov-2 presente no último ano possui muitas manifestações clínicas desde quadros influenza-like leves até síndrome respiratória aguda grave (SRAG). Algumas manifestações dermatológicas também se fazem presentes no quadro clínico. OBJETIVO: Realizar relato de caso de uma das manifestações dermatológicas da infecção pelo Covid-19, o Eritema Nodoso (EN). MÉTODO: Trata-se de um relato de caso, descritivo, baseado na clínica de uma paciente com manifestações iniciais de síndrome gripal, sendo confirmado via RTPCR infecção pelo SARS-Cov-2, evoluindo com quadro de EN. Os dados do relato foram obtidos pelo prontuário e exames laboratoriais. RESULTADO: As manifestações cutâneas apresentadas por pacientes portadores de infecções virais, como o Covid-19, são variadas e apresentam relação com tais patógenos. Estão relacionados à fisiopatologia fenômenos como reação de hipersensibilidade tipo IV, hipercoagulabilidade sanguínea e acometimento sistêmico culminando em exacerbada resposta inflamatória e produção de citocinas. Tal resposta orgânica pode levar a manifestações cutâneas, como o EN, o qual pode ser causado por outras etiologias descartadas durante a investigação do caso clínico relatado. CONCLUSÃO: Este artigo ao abordar outras manifestações da COVID-19, pode ser um meio de auxílio em futurosdiagnósticos desta doença. O EN, neste caso, foi após

\footnotetext{
${ }^{1}$ Médica graduada pela Universidade de Uberaba (UNIUBE), Residente do primeiro ano de clínica médica pelo Mário Palmério Hospital Universitário. mansurgeorgia@gmail.com.

${ }^{2}$ Médico graduado pela Universidade de Uberaba (UNIUBE). bernardo_88@hotmail.com.

${ }^{3}$ Médica graduada pela Universidade de Uberaba (UNIUBE), Residente do primeiro ano de Clínica Médica pelo Mário Palmério Hospital Universitário. aamandalivia@gmail.com.

${ }^{4}$ Médico graduado pela Universidade de Uberaba (UNIUBE), Residente do primeiro ano de Clínica Médica pelo Mário Palmério Hospital Universitário. tiagolucastadeu@hotmail.com.

${ }^{5}$ Médico graduado pela Universidade Federal de Minas Gerais (UFMG), Residente do primeiroano de Clínica Médica pelo Mário Palmério Hospital Universitário. diogolemos89@gmail.com.

${ }^{6}$ Médico graduado pela Universidade Federal do Triângulo Mineiro, Infectologista também pela Universidade Federal do Triângulo Mineiro (UFTM). guilhermehm7@hotmail.com.
} 
o diagnóstico de uma infecção viral, mas, nada impede, que em pacientes assintomáticos seja uma forma inicial de chegar a essa hipótese. Dessa forma, 0 relato tem como finalidade ampliar meios diagnósticos e expor a relação da corona vírus com demais manifestações clínicas.

Palavras chave: Pandemia. Infecções por coronavírus. Betacoronavírus. Eritema nodoso. Manifestações cutâneas.

ABSTRACT: The SARS-Cov-2 pandemic present in the last year has many clinical manifestations ranging from mild influenza-like conditions to severe acute respiratory syndrome (SARS). Some dermatological manifestations are also in the clinical picture. OBJECTIVE: Make a case report of one of the dermatological manifestations of infection by Covid-19, erythema nodosum (EN). METHOD: It is a descriptive case report, based on the clinic study of a patient with initial manifestations of influenza syndrome, which infection by SARS-Cov-2 was confirmed via RT-PCR, that evolved as a picture of erythema nodosum. Report data were obtained from medical records, including laboratory tests. RESULT: The skin manifestations presented by patients with viral infections, like Covid-19, can be varied and are related to such pathogens. They are associated to the pathophysiology phenomena such as type IV hypersensitivity reaction, blood hypercoagulability and systemic involvement culminating in an exacerbated inflammatory response and cytokine production. This organic response canlead to skin manifestations, just as erythema nodosum, which can be caused by other etiologies discarded during the investigation of the reported clinical case. CONCLUSION: This article, when addressing other COVID-19 manifestations can be an aid in other future diagnoses of this disease. The erythema nodosum in this case was after the diagnosis of a viral infection, but nothing prevents that in asymptomatic patients it could be an initial way of turning into this hypothesis. Thus, this report aims to expand diagnostic methods and expose the relationship between the coronavirus and other clinical manifestations.

KEYWORDS: Pandemic. Coronavirus infections. Betacoronavirus. Erythema nodosum. Skin manifestations. 


\section{INTRODUÇÃO}

Em Dezembro de 2019 uma nova variante do já conhecido Coronavírus emergiu na cidade de Wuhan, província de Hubei, na China. Trata-se de um betacoronavírus nomeado SARS-CoV-2 e responsável pela exponencial dissipação desta doença aoredor de todo o mundo, tornando-se, então, pandêmica. O quadro desta moléstia possui um espectro variado de manifestações, desde quadros assintomáticos ou oligossintomáticos até quadros severos, levando a necessidade de internações devido a SRAG, com necessidade de ventilação mecânica e, algumas vezes, culminando em desfechos desfavoráveis, como óbito do paciente.

Existe ainda uma gama de manifestações inespecíficas, que estão também relacionadas a outros diagnósticos diferencias. Podemos observar a presença de tosse, coriza, febre, odinofagia, cefaleia, mialgia, anosmia, aguesia, astenia, prostração, além de manifestações dermatológicas variadas.

Dentro das manifestações dermatológicas observamos a presença de rash cutâneo eritematoso, urticária, vesículas, tinea pedis, bem como ocorre em outras infeções virais. Neste relato de caso abordaremos a infecção pelo SARS-CoV-2 e a presença do eritema nodoso.

\section{MÉTODO}

O presente estudo é um relato de caso, descritivo, que retrata uma paciente do sexo feminino com clínica inicial de uma síndrome gripal e após três semanas houve evolução com quadro de Eritema Nodoso. A coleta de dados foi iniciada em Setembro de 2020 através do reconhecimento do caso clinico e provável correlação etiológica. Foram realizadas buscas em plataformas digitais como Pubmed, Jornais e Revistas Médicas relacionadas aos temas ertitema nodoso e manifestações 
cutâneas pelo Covid-19. Os exames laboratoriais para avaliação de diagnosticos diferenciais foram realizadosem Novembro de 2020.

- Identificação:

Trata-se de paciente do sexo feminino, 46 anos, IMC: 26,88, sedentária, professora, moradora da cidade de Conceição das Alagoas - MG. Nega hábitos etílicos, tabagismo ou uso de drogas ilícitas.

- Antecedentes pessoais:

Possui como antecedentes pessoais vitiligo, diagnosticado aos 2 anos de idade e enxaqueca, aos 6 anos. Faz uso diário de pregabalina $75 \mathrm{mg} 1$ comprimido a noite, propranolol $40 \mathrm{mg} 1$ comprimido pela manhã.

Como antecedentes cirúrgicos realizou mamoplastia aos 20 anos, cesárea aos 31 anos, cirurgia bariátrica aos 34 anos, abdominoplastia aos 40 anos, colecistectomia aos 42 anos.

Quanto à história obstétrica: G1C1A0, sendo cesárea realizada em 2006, após gestação sem intercorrência.

- História da doença atual:

Paciente iniciou quadro em Julho de 2020 apresentando tosse, coriza, anosmia, ageusia, cefaleia, febre com piora progressiva de tais sintomas, o que levou a buscar atendimento em pronto socorro na cidade de origem. Em atendimento inicial fora prescrito medicamentos sintomáticos, como analgésicos, antialérgicos, além de azitromicina $500 \mathrm{mg} 1$ comprimido ao dia por 5 dias, Ivermectina $6 \mathrm{mg}$, 2 comprimidos em dose única, dexametasona $6 \mathrm{mg}, 1$ comprimido ao dia. Realizado ainda afastamento das atividades usuais, além de isolamento domiciliar da paciente e contactantes domiciliares. Solicitado RT-PCR para Covid19 , via swab naso-oral, sendo resultado positivo para o agente. 
Houve melhora do quadro respiratório em 5 dias sem intercorrências drásticas ou necessidade de internação hospitalar, porém, houve persistência de aguesia e anosmia por 16 semanas.

Após 3 semanas do quadro clinico inicial houve presença de manifestações cutâneas, em região anterior de MMII, em especial na região pré-tibial. Estas manifestações caracterizavam-se pela presença de nodulações, com diâmetro entre 2 e $6 \mathrm{~cm}$, com formato ovoide e coloração violácea (Figura 1). Queixava-se ainda de dor local importante com progressivo aumento, o que impedia a realização de atividades diárias. Não houve queixas de prurido, sangramento, perda de sensibilidade, parestesias nas lesões. Não havia ulcerações ou pontos de necrose.

Figura 1 - Eritema Nodoso.

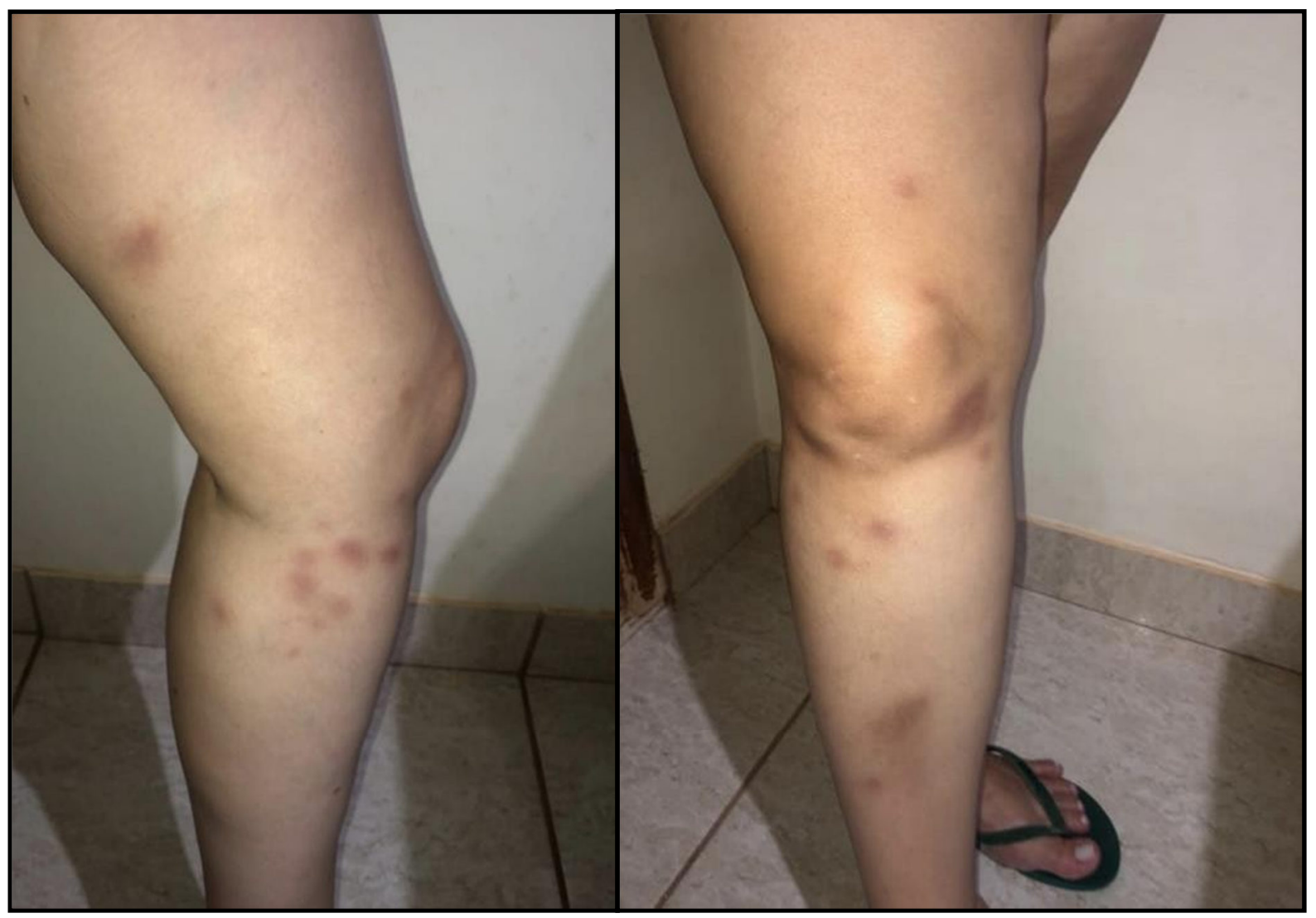

Fonte: Dos próprios autores, 2020.

Encaminhada para avaliação no Dermatologista, foi aventada a hipótese diagnóstica de eritema nodoso, uma inflamação do tecido subcutâneo (paniculite) 
consequência de reações de hipersensibilidade e que possui várias etiologias, sendo uma delas as infecções virais. A fim de prosseguir com investigação e descartar outras possíveis causas etiológicas para o eritema nodoso, foram realizados os seguintes exames laboratoriais, com os respectivos resultados: HbsAg não reagente, Anti-HBs não reagente, Anti HCV não reagente, Anti Hiv I e II não reagentes, VDRL não reagente, Sorologias para Rubeola IgM não reagente e IgG reagente, Epstein Barr IgM não reagente e IgG reagente, Citomegalovirus IgM não reagente e IgG reagente, Doença Chagas IgG não reagente, FAN Hep-2 não reagente. Prescrito em atendimento dermatológico Prednisona $20 \mathrm{mg}, 1$ comprimido ao dia, por 30 dias. Neste período do uso do medicamento proposto pelo dermatologista houve melhora importante de lesões pré-tibiais, porém, para total resolução do quadro foram ainda necessários 5 meses.

\section{DISCUSSÃO}

Schwartz et al. (2007) aponta que no eritema nodoso ocorre uma reação de hipersensibilidade tipo IV, com ativação de mastócitos e basófilos levando ao quadro depaniculite, isto é, uma inflamação do tecido subcutâneo gorduroso. Caracteriza-se por início agudo de nódulos eritematosos/violáceos, dolorosos, não supurativos, formato ovoide em especial na porção anterior de MMII, porém, podem acometer também membros superiores (MMSS), tronco e face. Em alguns casos coexistem sintomas sistêmicos como febre, edema MMII, artralgia, alterações gastrointestinais. Não é comum a presença de ulcerações, necrose ou presença de cicatrizes, entretanto, pode haver hiperpigmentação residual, o que é chamado de eritema contusiformis (Acosta et al - 2013), tornando-se crônico em 1/3 dos casos, sendo a duração maior que 6 meses. Sua etiologia é variada e compreende infecções virais, bacterianas, gravidez, malignidade, sarcoidose e, até mesmo, uso de medicações como contraceptivos orais.

O eritema nodoso é mais comum em pacientes do sexo feminino (6:1), na faixa etária entre 25 e 40 anos. Sua fisiopatogenia não é muito bem elucidada, 
porém, relaciona-se a ação da enzima conversora de angiotensina II (ECA-II), presente na camada basal da epiderme, como descrito por Hammining et al (2004). Há atuação também de uma protease trasmembrana chamada Serina, assim como ocorre em outras reações cutâneas, como urticária e púrpuras. Sabe-se ainda que devido reações de hipersensibilidade e ativação exacerbada de citocinas inflamatórias leva ao que chamamos de "tempestade de citocinas", como referido por Li et al.(2020).

Sabe-se que é uma das possíveis manifestações cutâneas que podem acometer indivíduos portadores de infecções virais (Tabela - 1 - Schwartz et al. 2007) e, portanto, encontra-se presente no amplo quadro clínico daqueles infectados pelo SARS-CoV-2.

Tabela 1 - Principais Causas de Eritema Nodoso - Schwartz et al. (2007).

\begin{tabular}{l} 
COMUNS \\
\hline Idiopático (> 55\%) \\
Infeções: faringite estreptocócica (28 - 48\%), Yersinia spp. \\
(Europa), mycoplasma, chlamydia, histoplasmosis, \\
coccidioidomycosis, mycobacteria \\
\hline Sarcoidose (11 - 25\%) \\
\hline Fármacos: antibióticos (sulfonamidas, amoxicilina), \\
contracetivos orais ( 3 - 10\%) \\
Gravidez (2 - 5\%) \\
\hline Doença inflamatória intestinal: Doença de Crohn, colite \\
ulcerosa (1 - 4\%) \\
\hline RARAS (< 1\%) \\
Infeções \\
- Virais: vírus herpes simples, vírus Epstein-Barr, vírus \\
hepatite B e C, virus da imunodeficiência humana \\
- Bacterianas: Campylobacter spp., rickettsiae, Salmonella \\
spp., psittacosis, Bartonella spp., syphilis \\
- Parasitárias: amebíase, giardíase \\
\hline Outras: linfomas, outras patologias malignas
\end{tabular}

Fonte: Disponível em: Dossier (mec.pt). Acesso em: 27 Out. 2020. 
Neste relato de caso, devido ao recente diagnostico confirmado para Covid-19 e tendo em vista que outras possíveis causas para desenvolvimento do eritema nodoso foram excluídas, além da relação entre tal manifestação cutânea e infecções virais, pode-se inferir que tal diagnostico dermatológico, é o Eritema Nodoso. Além disso, sabe-se que o diagnóstico de tal comorbidade é clinico.

A terapêutica inicial deve-se concentrar no tratamento da doença de base, sendo no caso do Covid-19, tratamentos sintomáticos, uma vez que ainda não há terapêutica especifica definitiva. Além disso, deve-se lançar mão de medicamentos também sintomáticos, como analgésicos simples, anti-inflamatórios não esteroidais (AINES), corticoides sistêmicos, repouso e, até mesmo, meias compressivas para os casos álgicos refratários,- Schwartz et al. (2007). Sabe-se que é um acometimento cutâneo benigno e autolimitado na maior parte dos casos, com melhora total em dias ou poucas semanas.

\section{CONCLUSÃO}

Diante de dias como os vivenciados no último ano devido ao acometimento global pelo SARS-CoV-2 e seus desdobramentos clínicos variados e multissistêmicos, pode-se concluir que ainda serão observadas diversas associações a este mesmo agente etiológico, uma vez que mesmo o Corona vírus seja conhecido há anos, esta nova cepa ainda é recente para afirmações contundentes em algumas áreas da medicina e, portanto, faz necessário mais pesquisas, relatos de casos, incremento da literatura. Sabe-se, ainda, que a melhor maneira para conter a pandemia e promover diminuição da incidência de casos, bem como suas manifestações dermatológicas é adotar medidas preventivas de disseminação viral, sendo elas: lavar frequentemente as mãos com sabão ou fazer uso do álcool gel, manter isolamento e distanciamento social, usar máscaras de forma correta e efetiva e, recentemente, a vacinação. 
Em suma, este relato de caso retrata uma paciente do sexo feminino que 3 semanas após manifestações de uma síndrome gripal inicial, sendo confirmado diagnóstico de Covid-19, desenvolveu quadro de Eritema Nodoso. Dessa maneira, é relevante que saibamos as possíveis manifestações que ultrapassam o quadro clínico clássico de acometimento do sistema respiratório, para que, de modo gradativo, possamos acumular informações relevantes sobre essa doença e elucidar melhor seu quadro clínico e desdobramentos.

Este artigo ao abordar outras manifestações da COVID-19, pode ser um meio de auxílio em futuros diagnósticos desta doença. O EN, neste caso, foi após o diagnóstico de uma infecção viral, mas, nada impede, que em pacientes assintomáticos seja uma forma inicial de chegar a essa hipótese. Dessa forma, o relato tem como finalidade ampliar meios diagnósticos e expor a relação da corona vírus com demais manifestações clínicas, além disso, com maior nível de conhecimento e suspeição clínica haverá ênfase na prevenção quaternária aos pacientes, evitando possíveis iatrogenias, como no própriocaso do eritema nodoso, que na maior parte das vezes é um desdobramento benigno e autolimitado.

\section{REFERÊNCIAS BIBLIOGRÁFICAS}

ACOSTA, K.A., HAVER, M.C. \& KELLY, B. Etiology and Therapeutic Management of Erythema Nodosum During Pregnancy: An Update. Am J Clin Dermatol 14, 215- 222 (2013). Disponível em: https://doi.org/10.1007/s40257-013-0024-x Acesso em: 03 Fev. 2021.

CHEN, Y., LIU, Q., GUO, D. Emerging coronaviruses: genome structure, replication, and pathogenesis. J Med Virol. 2020;92(4):418-23. Disponível em: Emerging coronaviruses: Genome structure, replication, and pathogenesis - PubMed (nih.gov) Acesso em: 30 Jan. 2021

CRIADO, P.R et al. Are the cutaneous manifestations during or due to SARS-CoV-2 infection/COVID-19 frequent or not? Revision of possible pathophysiologic mechanisms. Inflamm. Res. 69, 745-756 (2020). Disponível em: Are the cutaneous manifestations during or due to SARS-CoV-2 infection/COVID-19 frequent or not? Revision of possible pathophysiologic mechanisms - PubMed (nih.gov). Acesso em: 30Jan. 2021.

CRIBIER, B., CAILLE, A., HEID, E., et al. Erythema nodosum and associated diseases: A study of 129 cases. Int J Dermatol 1998;37:667-72. Disponível em: Erythema nodosum and associated diseases. A study of 129 cases - Cribier - 1998 - International Journal of Dermatology - Wiley Online Library Acesso em: 30 Jan. 2021.

ESTÉBANEZ, A., PÉREZ-SANTIAGO, L., SILVA, E., et al. Cutaneous manifestations in COVID- 19:a new contribution. J Eur Acad Dermatol Venereol 2020;34:e250-1. Disponível em: 
"https://www.ncbi.nlm.nih.gov/pmc/articles/PMC7262266/pdf/JDV-9999-na.pdf"\& HYPERLINK "https://www.ncbi.nlm.nih.gov/pmc/articles/PMC7262266/pdf/JDV- 9999-na.pdf"\#x02010;19: a new contribution (nih.gov) Acesso em: 29 Jan. 2021.

FERRO, F., et al. COVID-19: the new challenge for rheumatologists. Clin Exp Rheumatol. 2020;38(2):175-80. Disponível em: COVID-19: the new challenge for rheumatologists (clinexprheumatol.org). Acesso em: 28 Jan. 2021.

FOX, M. D., SCHWARTZ, R. A. Erythema nodosum. Am Fam Physician. 1992 Sep;46(3):81822. PMID: 1514475. Disponível em: https://pubmed.ncbi.nlm.nih.gov/1514475/ Acesso em: 03 Fev. 2021

HAMMING, I., et al. Tissue distribution of ACE2 protein, the functional receptor forSARS coronavirus. A first step in understanding SARS pathogenesis. J Pathol. 2004;203(2):6317. Disponivel em: Tissue distribution of ACE2 protein, the functional receptor for SARS coronavirus. A first step in understanding SARS pathogenesis - Hamming - 2004 - The Journal of Pathology - Wiley Online Library. Acesso em: 02 Fev. 2021.

JIN Y-H, LIN C, CHENG Z-S, et al. A rapid advice guideline for the diagnosis and treatment of 2019 novel coronavirus (2019-nCoV) infected pneumonia (standard version). Mil Med Res. 2020;7:4. Disponível em: A rapid advice guideline for the diagnosis and treatment of 2019 novel coronavirus (2019-nCoV) infected pneumonia (standard version) | Military Medical Research | Full Text (biomedcentral.com). Acessoem: 02 Fev. 2021.

LI, G., FAN, Y., LAI, Y., et al. Coronavirus infections and immune responses. J Med Virol. 2020;92:424-432.|LI ET AL. Disponível em: Coronavirus infections and immuneresponses - Li 2020 - Journal of Medical Virology - Wiley Online Library. Acesso em:02 Fev. 2021.

MANALO, I. F., SMITH, M. K., CHEELEY, J., et al. A dermatologic manifestation of COVID19:transient Livedo reticularis. J Am Acad Dermatol 2020:30558-2. Disponível em: A dermatologic manifestation of COVID-19: Transient livedo reticularis - Journal of the American Academy of Dermatology (jaad.org). Acesso em: 01 Fev. 2021.

PROMPETCHARA, E., KETLOY, C., PALAGA, T. Immune responses in COVID-19 and potential vaccines: lessons learned from SARS and MERS epidemic. Asian Pac J Allergy Immunol. 2020;38(1):1-9. Disponível em: 1.pdf (apjai-journal.org). Acesso em: 01 Fev.2021.

RECALCATI, S. Cutaneous manifestations in COVID-19: a first perspective. J Eur Acad Dermatol Venereol. 2020 May;34(5):e212-e213. doi: 10.1111/jdv.16387. PMID: 32215952. Disponivel em: https://onlinelibrary.wiley.com/doi/epdf/10.1111/jdv.163. Acesso em: $01 \mathrm{Fev}$. 2021.

REQUENA, L., SÁNCHEZ YUS, E. Erythema nodosum. Semin Cutan Med Surg. 2007 Jun;26(2):114-25. doi: 10.1016/j.sder.2007.02.009. PMID: 17544964. Disponível em: Erythema Nodosum - SCMSJournal.com. Acesso em: 01 Fev. 2021.

SCHWARTZ, R. A., NERVI, S. J. Erythema nodosum: a sign of systemic disease. Am Fam Physician. 2007 Mar 1;75(5):695-700. PMID: 17375516. Disponível em: Erythema Nodosum: A Sign of Systemic Disease - American Family Physician (aafp.org). Acessoem: 01 Fev. 2021.

SUTER, P., MOOSER, B., PHAM HUU THIEN, H. P. Erythema nodosum as a cutaneous manifestation of COVID-19 infection. BMJ Case Rep 2020;13:e236613doi:10.1136/bcr 2020236613. Disponível em: http://casereports.bmj.com/. Acesso em: 27 Out. 2020.

$\mathrm{TAO}$, J., et al. Emergency management for preventing and controlling nosocomial infection of 2019 novel coronavirus: implications for the dermatology department. $\mathrm{Br} \mathrm{J}$ Dermatol 2020. Disponível em: Emergency management for preventing and controlling 
nosocomial infection of the 2019 novel coronavirus: implications for the dermatology department - Tao - 2020 - British Journal of Dermatology - Wiley OnlineLibrary. Acesso em: 02 Fev. 2021.

TÜRSEN, Ü., TÜRSEN, B., LOTTI, T. Coronavirus-days in dermatology. Dermatologic Therapy. 2020; 33:e13438. Disponível em: Coronavirus- days in dermatology (wiley.com) Acesso em: 02 Fev. 2021. 